hep-th/0510098

\title{
Thermodynamics of the Near-Extremal NS5-brane
}

\author{
Troels Harmark and Niels A. Obers \\ The Niels Bohr Institute \\ Blegdamsvej 17, 2100 Copenhagen Ø, Denmark \\ harmark@nbi.dk, obers@nbi.dk
}

\begin{abstract}
We consider the thermodynamics of the near-extremal NS5-brane in type IIA string theory. The central tool we use is to map phases of six-dimensional Kaluza-Klein black holes to phases of near-extremal M5-branes with a transverse circle in eleven-dimensional supergravity. By S-duality these phases correspond to phases of the near-extremal type IIA NS5-brane. One of our main results is that in the canonical ensemble the usual nearextremal NS5-brane background, dual to a uniformly smeared near-extremal M5-brane, is subdominant to a new background of near-extremal M5-branes localized on the transverse circle. This new stable phase has a limiting temperature, which lies above the Hagedorn temperature of the usual NS5-brane phase. We discuss the limiting temperature and compare the different behavior of the NS5-brane in the canonical and microcanonical ensembles. We also briefly comment on the thermodynamics of near-extremal $\mathrm{D} p$-branes on a transverse circle.
\end{abstract}




\section{Introduction}

The physics of the NS5-brane is interesting for many reasons: It is conjectured to have a novel non-gravitational string theory living on its world-volume [1, 2, 3]. The type IIA NS5-brane world-volume theory has the $(2,0)$ super conformal field theory as its low energy limit 4]. Moreover, the NS5-brane wrapped on certain cycles describes pure super Yang-Mills theory [5].

In this paper we consider the thermodynamics of the near-extremal NS5-brane in type IIA string theory. We use as a starting point that the type IIA NS5-brane is given nonperturbatively as an M5-brane with a transverse circle. More specifically, we employ the idea of [6, 7] that in order to understand the thermodynamic phases of the type IIA NS5brane it is necessary to know all possible phases of M5-branes with a transverse circle. ${ }^{1}$

An important aspect of the NS5-brane is that the thermodynamics of the usual nearextremal NS5-brane background (dual to an M5-brane uniformly smeared on a transverse circle) has entropy as function of energy $S(E)=T_{\mathrm{hg}}^{-1} E$, where $T_{\mathrm{hg}}$ is a fixed temperature [8. 9. Thus, the thermodynamics of the NS5-brane is singular and has Hagedorn-like behavior, supporting the idea that its non-gravitational dual is a string theory. In [10, 11] the idea was put forward that the singular thermodynamics of the NS5-brane could be resolved by computing a string one-loop correction to the background. This was done in [12], with the result that the corrected background has $T>T_{\mathrm{hg}}$ and negative specific heat. In a subsequent analysis the results of [12] have been interpreted to indicate that one does not have a Hagedorn phase transition and that the Hagedorn temperature is a limiting temperature [13].

The main result of this paper is that in the canonical ensemble the usual near-extremal NS5-brane background is thermodynamically subdominant to a new eleven-dimensional background of near-extremal M5-branes localized on the transverse circle. This means that if one starts with the usual near-extremal NS5-brane background this will decay and end up in the new phase which is thermodynamically stable in that it has a positive specific heat. That the new phase is dominant means in particular that the string one-loop correction, or any other correction to the usual near-extremal NS5-brane background, is subdominant to the effect of the decay to the new eleven-dimensional phase. The new phase is also dominant in the microcanonical ensemble for sufficiently small energies.

However, the presence of the new stable phase also raise a puzzle. The puzzle origins in the fact that the new phases we discuss in this paper have a maximally possible temperature. Therefore, it is not clear what happens if one tries to heat up the type IIA NS5-brane to a temperature higher than this maximal temperature. As we discuss in the paper, this is also connected to the fact that the canonical and microcanical ensembles

\footnotetext{
${ }^{1}$ In this paper we consider only the near-extremal type IIA NS5-brane, but our results can easily be extended to the full non-extremal case. However, we restrict ourselves to the near-extremal case since this has the most interesting physics and since it is dual to a decoupled non-gravitational theory.
} 
seem to be inequivalent at large energies.

The new results in this paper on the thermodynamics of the NS5-brane are obtained using the transformation of [6] 1] that can take a phase of a six-dimensional Kaluza-Klein black holes and transform it into a phase for near-extremal M5-branes with a transverse circle. Our focus in this paper is on phases without Kaluza-Klein bubbles since these are the dominant phases in the phase diagram. Phases obtained from the solutions with Kaluza-Klein bubbles in [14] are considered in [15].

For comparison, we also briefly discuss the thermodynamics of near-extremal $\mathrm{D} p$-branes on a circle. These cases have some properties in common with the M5-brane case, but also differ significantly in some other aspects. In particular, they do neither exhibit a maximal temperature, nor a discrepancy between the microcanonical and canonical ensembles.

The outline of this paper is as follows. In Section 2 we review some of the basic facts on near-extremal NS5-branes that we build on in the rest of the paper. In Section [3 we review how one maps phases of six-dimensional Kaluza-Klein black holes to phases of near-extremal type IIA NS5-branes, and we use recently obtained data to find the entire localized phase. In Section 4 we discuss the new thermodynamics of the near-extremal type IIA NS5-brane in the canonical and microcanonical ensembles. In Section 5 we comment on similarities in the thermodynamics of near-extremal $\mathrm{D} p$-branes on a transverse circle. Finally, we present our conclusions in Section [6]

\section{Basic facts on near-extremal NS5-branes}

In this section we briefly review basic facts about near-extremal NS5-branes. Note that the results of [6, 7] for near-extremal NS5-brane on which we build in this paper, are not reviewed in this section but instead in Sections 3 and 4 .

One of the reasons why the thermodynamics of near-extremal NS5-branes is interesting is the fact that this background is believed to be dual to a non-gravitational theory. In the decoupling limit of $N$ coincident NS5-branes in type IIA string theory the string length $l_{s}$ is kept fixed while the string coupling $g_{s}$ goes to zero [9, 16]. In this limit the dynamics of the theory is believed to reduce to a string theory without gravity, called Little String Theory (LST), or more precisely $(2,0)$ LST of type $A_{N-1}$ [1, 2, 3]. ${ }^{2}$

Consider now instead $N$ non-extremal type IIA NS5-branes. Then the decoupling limit, or near-extremal limit, is defined as keeping $l_{s}$ fixed and taking $g_{s}$ to zero, while at the same time keeping the energy above extremality fixed. This gives the following

\footnotetext{
${ }^{2}$ See [17] [18] 19] 20], 21, 22, [3] 23] for other interesting work on thermodynamics and correlators of Little String Theory.
} 
background (in the string frame) describing $N$ coincident near-extremal NS5-branes ${ }^{3}$

$$
\begin{gathered}
d s^{2}=-f d t^{2}+\sum_{i=1}^{5} d x_{i}^{2}+\frac{N l_{s}^{2}}{r^{2}} f^{-1} d r^{2}+N l_{s}^{2} d \Omega_{3}^{2}, \quad f=1-\frac{r_{0}^{2}}{r^{2}} \\
g_{s}^{2} e^{2 \phi}=\frac{N}{r^{2}}, \quad H_{3}=d B_{2}=2 N l_{s}^{2} d \Omega_{3} .
\end{gathered}
$$

Here $\phi$ is the dilaton and $B_{2}$ is the Kalb-Ramond two-form potential for the field strength $H_{3}$ under which NS5-branes are magnetically charged. The thermodynamics of this background is

$$
T=T_{\mathrm{hg}} \equiv \frac{1}{2 \pi \sqrt{N} l_{s}}, \quad S(E)=T_{\mathrm{hg}}^{-1} E, \quad \frac{E}{V_{5}}=\frac{r_{0}^{2}}{(2 \pi)^{5} l_{s}^{6}} .
$$

This thermodynamics corresponds to the thermodynamic behavior of a string theory at the Hagedorn temperature, $T_{\text {hg }}$ being the Hagedorn temperature [8, 9]. The NS5-brane description of $(2,0) \mathrm{LST}$ is valid at high energies $E / V_{5} \gg \mathrm{Nl}_{s}^{-6}$.

The above background corresponds to the exact worldsheet CFT given by $H_{3}^{+} / U(1) \times$ $S U(2)_{N} \times \mathbb{R}^{5}$, with $H_{3}^{+} \equiv S L(2, \mathbb{C})_{N} / S U(2)_{N}$, which can be used to perform string computations in this background. In particular, the string coupling expansion in the background becomes an expansion around infinite energy in powers of $1 / E[24]$. Thus, one expects the stringy features of the world-volume theory of NS5-branes to appear in the high energy regime $E / V_{5} \gg N l_{s}^{-6}$.

As mentioned in the Introduction it was suggested in [10, 11] that one could resolve the singular thermodynamics of the near-extremal NS5-brane by computing string corrections to the NS5-brane background. In [12] this was done using the fact that the near-extremal NS5-brane background is described by an exact worldsheet CFT. The result of this computation is that the corrected background has $T>T_{\mathrm{hg}}, F>0$ and negative specific heat. Subsequently, the instability of this background was argued in 13] to be a tad-pole instability since the unstable mode has a potential proportional to $-\log E$, driving the mode to larger values of the energy. See the conclusions in Section 6 for further comments on the high energy regime and comparison to the results of this paper.

Turning to lower energies, we have that using the IIA/M S-duality the near-extremal NS5-brane can be seen as an M5-brane in M-theory which is smeared uniformly in a transverse direction. The background (2.1) is therefore equivalent to the following Mtheory background

$$
\begin{gathered}
l_{p}^{-2} d s^{2}=\frac{r^{2 / 3}}{N^{1 / 3} l_{s}^{2}}\left[-f d t^{2}+\sum_{i=1}^{5} d x_{i}^{2}\right]+\frac{N^{2 / 3}}{r^{4 / 3}}\left[f^{-1} d r^{2}+d z^{2}+r^{2} d \Omega_{3}^{2}\right] \\
F_{4}=d C_{3}=2 N l_{p}^{3} d z d \Omega_{3} .
\end{gathered}
$$

where $f$ is the same as given in (2.1) and $C_{3}$ is the three-form potential for the fourform field strength under which the M5-brane is magnetically charged. This background

\footnotetext{
${ }^{3}$ Note that the radial coordinate $r$ is defined to be dimensionless in (2.1).
} 
describes $N$ coincident near-extremal M5-branes smeared on a transverse circle. The transverse circle is parameterized by $z$ which we take to have period $2 \pi$. The background (2.3) is weakly curved when $N \gg 1$. The near-extremal limit of non-extremal M5-branes with a transverse circle is given by taking $l_{p} \rightarrow 0$ while rescaling the transverse directions. ${ }^{4}$ The thermodynamics is identical to that of the near-extremal NS5-brane given above, and this description of $(2,0)$ LST is valid for energies $l_{s}^{-6} \ll E / V_{5} \ll N l_{s}^{-6}$.

At low energy the background of near-extremal M5-branes smeared on a transverse direction is unstable to decay to a background of near-extremal M5-branes localized on the transverse circle [7]. For very low energies the $N$ near-extremal NS5-branes thus enter a phase which is well-described as $N$ near-extremal M5-branes in eleven dimensional flat space. The dual theory of this background is the $(2,0)$ super conformal field theory (SCFT). We shall return to this in the following.

\section{$3 \quad$ NS5-brane phases from Kaluza-Klein black holes}

In this section we briefly review the map of $[6,7]^{5}$ by which one can obtain solutions for near-extremal M5-branes with a transverse circle from phases of Kaluza-Klein black holes in six dimensions. By six-dimensional Kaluza-Klein black holes we mean pure gravity solutions of six-dimensional General Relativity that at asymptotic infinity have the geometry of Kaluza-Klein space-time $\mathcal{M}^{5} \times S^{1}, \mathcal{M}^{5}$ being five-dimensional Minkowski space (see the reviews [27, 28] for more on Kaluza-Klein black holes). As we discuss below, the near-extremal M5-brane phases can be seen as phases of near-extremal type IIA NS5-branes.

As described in [14, 27] there are two classes of Kaluza-Klein black holes in six dimensions: Solutions with a local $S 0(4)$ symmetry, and solutions without a local $S O(4)$ symmetry. The latter we shall briefly discuss in the Conclusions, and these are ones that contain Kaluza-Klein bubbles. For the former class of solutions, which do have a local $S O(4)$ symmetry and which are the ones of interest in this paper, it has been proven [29, 30] that the metric can be put in the ansatz [6]

$$
d s^{2}=-f d t^{2}+\frac{A}{f} d R^{2}+\frac{A}{K^{3}} d v^{2}+K R^{2} d \Omega_{3}^{2}, \quad f=1-\frac{R_{0}^{2}}{R^{2}} .
$$

Here $A(R, v)$ and $K(r, v)$ are two functions determining the metric. $R \rightarrow \infty$ is the asymptotic region where $A$ and $K$ go to one, while $R=R_{0}$ is the event horizon [6]. We have $v \equiv v+2 \pi$, i.e. we take the asymptotic circle to have unit radius for simplicity.

Using the map found in [6, 7] we can now take any six-dimensional Kaluza-Klein black hole solution of the form (3.1) and transform it to a solution of eleven dimensional

\footnotetext{
${ }^{4}$ The near-extremal limit of the NS5-brane and the one of the smeared M5-brane are easily related using the S-duality relations $l_{p}^{3}=g_{s} l_{s}^{3}$ and $R_{11}=g_{s} l_{s}$ where $R_{11}$ is the radius of the eleventh direction which in this case is the transverse circle that the M5-branes are smeared on.

${ }^{5}$ See [25] 26] for reviews.
} 
supergravity for $N$ near-extremal M5-branes with a transverse circle given by [ 6 , 7 ]

$$
\begin{gathered}
l_{p}^{-2} d s^{2}=\frac{R^{2 / 3}}{N^{1 / 3} l_{s}^{2}}\left[-f d t^{2}+\sum_{i=1}^{5} d x_{i}^{2}\right]+\frac{N^{2 / 3}}{R^{4 / 3}}\left[\frac{A}{f} d R^{2}+\frac{A}{K^{3}} d v^{2}+K R^{2} d \Omega_{3}^{2}\right], \\
F_{4}=d C_{3}=2 N l_{p}^{3} d z d \Omega_{3} .
\end{gathered}
$$

Here $f$ is as in (3.1). The supergravity solution (3.2) is valid as long as $N \gg 1$, since this guaranties the geometry to be weakly curved. Note that in the near-extremal limit $l_{p} \rightarrow 0$ while $l_{s}$ is finite. The relation between the Planck length $l_{p}$ and the string length $l_{s}$ is explained in the previous section. See [6, 7] for more on the near-extremal limit of M5-branes with a transverse circle.

The important point is now that the type IIA NS5-brane is non-perturbatively defined as an M5-brane with a transverse circle. Therefore, the phases of $N$ coincident nearextremal type IIA NS5-branes are in fact the phases of $N$ coincident M5-branes with a transverse circle. We can thus get insight into the thermodynamics of near-extremal NS5branes by examining near-extremal M5-branes with a transverse circle as obtained from phases of six-dimensional Kaluza-Klein black hole solutions.

The relevant physical quantities for the near-extremal M5-brane solutions with a transverse circle of the form (3.2) are given as $[6,7]^{6}$

$$
E=\frac{V_{5} R_{0}^{2}(1-\chi)}{(2 \pi)^{5} l_{s}^{6}}, \quad r=\frac{1-6 \chi}{2-2 \chi}, \quad T=\frac{1}{2 \pi \sqrt{N} l_{s} \sqrt{A_{h}}}, \quad S=\frac{V_{5} \sqrt{N} R_{0}^{2} \sqrt{A_{h}}}{(2 \pi)^{4} l_{s}^{5}}
$$

where $E$ is the energy, $r$ is the relative tension (see [31, 7] for definition), $T$ is the temperature and $S$ is the entropy. We define here the number $\chi$ for a given solution by $K(R, v)=1-\chi R_{0}^{2} / R^{2}+\cdots$ in the asymptotic region $R \rightarrow \infty$, and $A_{h}$ is the value of $A$ on the horizon: $A_{h}=\left.A(R, v)\right|_{R=R_{0}}$. We can then relate the physical quantities (3.3) for nearextremal M5-branes with a transverse circle to the physical quantities of the corresponding six-dimensional Kaluza-Klein black hole by the map [7]

$$
\frac{E}{V_{5}}=\frac{5+n}{8} \frac{\mu}{(2 \pi)^{5} l_{s}^{6}}, \quad r=\frac{8 n}{5+n}, \quad T=T_{\mathrm{hg}} \mathfrak{t} \sqrt{\mathfrak{t} \mathfrak{s}}, \quad \frac{S}{V_{5}}=\frac{1}{(2 \pi)^{5} l_{s}^{6} T_{\mathrm{hg}}} \frac{\mathfrak{s}}{\sqrt{\mathfrak{t s}}} .
$$

Here $\mu$ is the rescaled dimensionless mass, $n$ is the relative tension, $\mathfrak{t}$ is the rescaled dimensionless temperature and $\mathfrak{s}$ is the rescaled entropy of the six-dimensional KaluzaKlein black hole, as defined in [32, 27]. ${ }^{7}$

We now consider the solutions for $N$ near-extremal M5-branes with a transverse circle obtained from transforming six-dimensional Kaluza-Klein black hole solutions with local $S O(4)$ symmetry, via the map from (3.1) to (3.2). The Kaluza-Klein black holes with local

\footnotetext{
${ }^{6}$ Note that these relations imply the Smarr formula $5 T S=2(3-r) E[7]$.

${ }^{7}$ For six-dimensional Kaluza-Klein black hole solutions with metric of the form (3.1) we have [6] 27.

$$
\mu=R_{0}^{2}\left[\frac{3}{2}-\chi\right], \quad n=\frac{1-6 \chi}{3-2 \chi}, \quad \mathfrak{t}=\frac{1}{\sqrt{A_{h}} R_{0}}, \quad \mathfrak{s}=\sqrt{A_{h}} R_{0}^{3} .
$$
}


$S O(4)$ symmetry come in three branches: The uniform phase, the non-uniform phase and the localized phase. We therefore naturally obtain the following three phases for $N$ nearextremal type IIA NS5-brane, i.e. for $N$ near-extremal M5-branes with a transverse circle 7]:

- Uniform phase. This phase corresponds to $N$ near-extremal M5-branes uniformly smeared on a transverse circle. This is the background given by (2.3). As discussed in Section 2 this background corresponds to the usual near-extremal NS5-brane background given by (2.1). The relative tension is $r=1 / 2$ for the uniform phase. Note that from the six-dimensional Kaluza-Klein black hole point of view the uniform phase corresponds to the uniform black string phase.

- Non-uniform phase. The non-uniform phase corresponds to the new phase discussed in Ref. [7] of near-extremal M5-branes non-uniformly distributed on the transverse circle. The non-uniform phase emanates from the uniform phase at the critical energy $^{8} E_{\mathrm{c}} / V_{5}=1.54 \cdot(2 \pi)^{-5} l_{s}^{-6}$. The slope in the $(E, r)$ diagram (Figure 1) at this point is given by $r(E) \simeq 1 / 2-0.39 \cdot(2 \pi)^{5} l_{s}^{6}\left(E-E_{\mathrm{c}}\right) / V_{5}$ [7]. Note that the non-uniform phase is mapped from the neutral non-uniform black string phase in six dimensions. The solutions for the neutral non-uniform black string were obtained numerically in 33 and the behavior near the Gregory-Laflamme mass was found in 34 .

- Localized phase. The localized phase corresponds to a background of near-extremal M5-branes localized on the transverse circle. For $E \rightarrow 0$ we have that $r \rightarrow 0$ corresponding to the fact that the background is well-described by near-extremal M5branes in eleven dimensional flat space (this background is dual to $(2,0)$ SCFT). The first correction to the background due to the presence of the transverse circle was obtained in Ref. [7] using the analytical results on neutral small black holes on cylinders of [35] (see also [36, 37]). The slope of the curve for the localized phase at the origin is given by $r(E) \simeq\left[9 \zeta(3) /\left(25 \pi^{2}\right)\right](2 \pi)^{5} l_{s}^{6} E / V_{5}[7$. Beyond the analytical results for small energies, the localized phase is known numerically by mapping the numerical solutions for localized black holes in six-dimensional Kaluza-Klein space obtained in Ref. [38. These newly obtained data is in fact what enables us in this paper to gain new insights into the thermodynamics of the NS5-brane, as we shall see in Section 4

We have depicted the three phases ${ }^{9}$ in an $(E, r)$ diagram in Figure 1. Note that part of the phase diagram for the near-extremal type IIA NS5-brane was obtained in [7, but here

\footnotetext{
${ }^{8}$ Note that the critical energy is obtained from the neutral Gregory-Laflamme mass using the map (3.4).

${ }^{9}$ Note that for the non-uniform and localized phase there are extra phases in the form of copies since the solutions can be copied $k$ times on the circle 39 30 7. At the level of solutions this means that given the solution (3.2) we obtain for any $k=2,3, \ldots$ a new solution with $A^{\prime}(R, v)=A(k R, k v), K^{\prime}(R, v)=$ $K(k R, k v)$ and $R_{0}^{\prime}=R_{0} / k$. This solution will have the physical quantities $E^{\prime}=E / k^{2}, r^{\prime}=r$.
} 
we add the extra numerical data of 38.

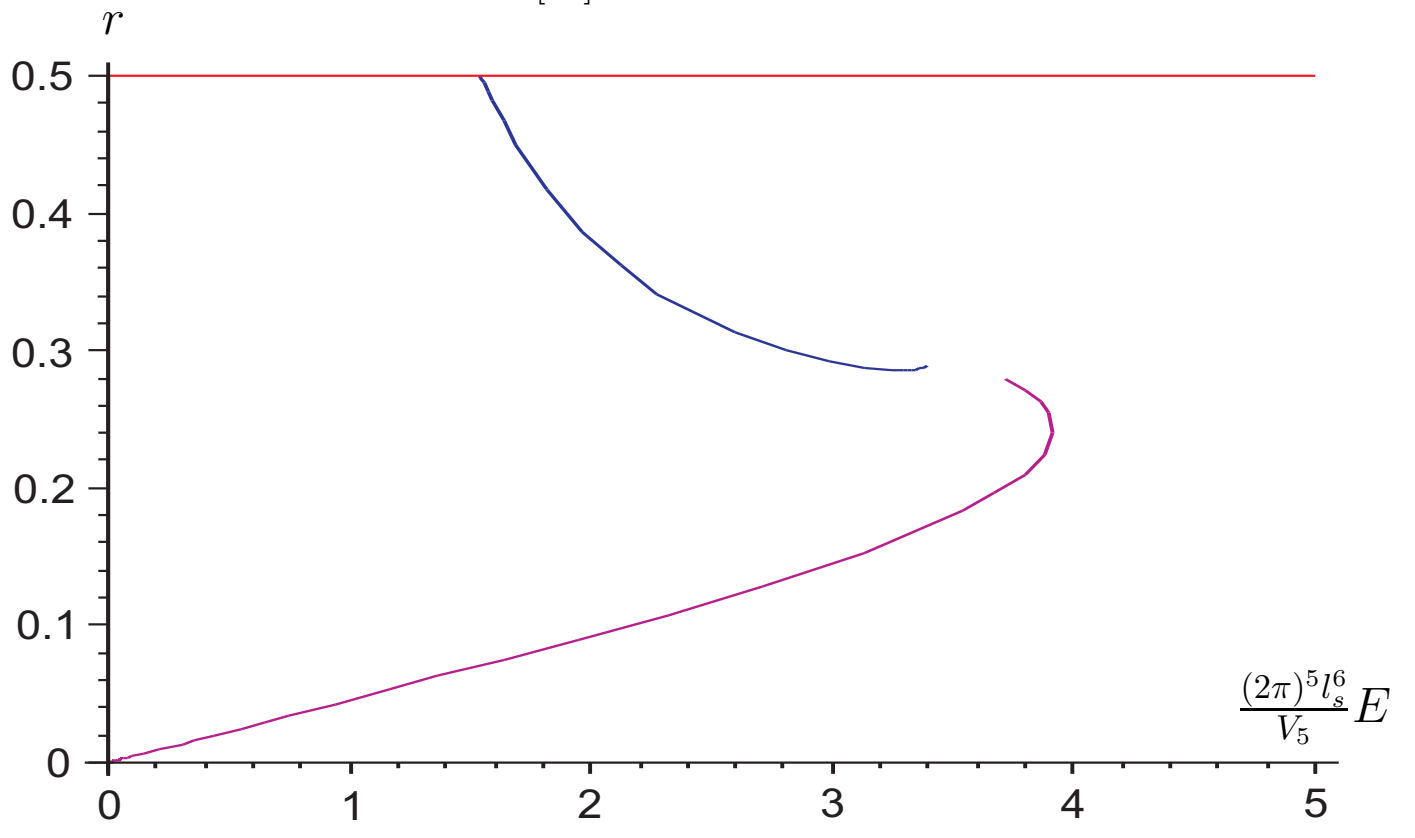

Figure 1: Relative tension versus energy for the uniform (red), non-uniform (blue) and localized (magenta) phase of near-extremal NS5-branes.

The uniform and non-uniform phase have horizon topology $R^{5} \times S^{3} \times S^{1}$, while the localized phase has $R^{5} \times S^{4}$. The $(E, r)$ diagram in Fig. 1 suggests that the non-uniform and localized phase meet each other in a topology changing transition. In fact, from the neutral Kaluza-Klein black hole point of view, Ref. 38 presented compelling evidence that the two branches indeed are meeting by examining various physical quantities of the numerical solutions.

The uniform phase of near-extremal NS5-branes is classically unstable for energies below the critical energy $E_{\mathrm{c}}$ 40, 41, 7, 42, 43]. In this regime the background is expected to decay to the localized phase corresponding to near-extremal M5-branes localized on a circle. Seen from type IIA String Theory this is a non-perturbative phenomenon since it involves localization on the M-theory circle.

It is important to note that the non-uniform and localized phases have non-singular event horizons. This one can see from i) the fact that the map from (3.1) to (3.2) takes a neutral solution with non-singular horizon to a near-extremal solution with non-singular horizon and ii) that it is known from the numerical results of [33, 38, that the neutral non-uniform black string and localized black hole phases have non-singular horizon. This can also be seen from (3.2) directly, by noting that the horizon is non-singular if $A(R, v)$ and $K(R, v)$ are well-behaved for $R \rightarrow R_{0}$. 


\section{New thermodynamics of the NS5-brane}

In this section we describe the thermodynamics of the near-extremal NS5-brane using the input of the new phases obtained from six-dimensional Kaluza-Klein black holes, as discussed in Section 3

\section{Canonical ensemble}

We begin with the canonical ensemble since this is the one with the most interesting results. We have displayed the free energy versus the temperature in Fig. 2.

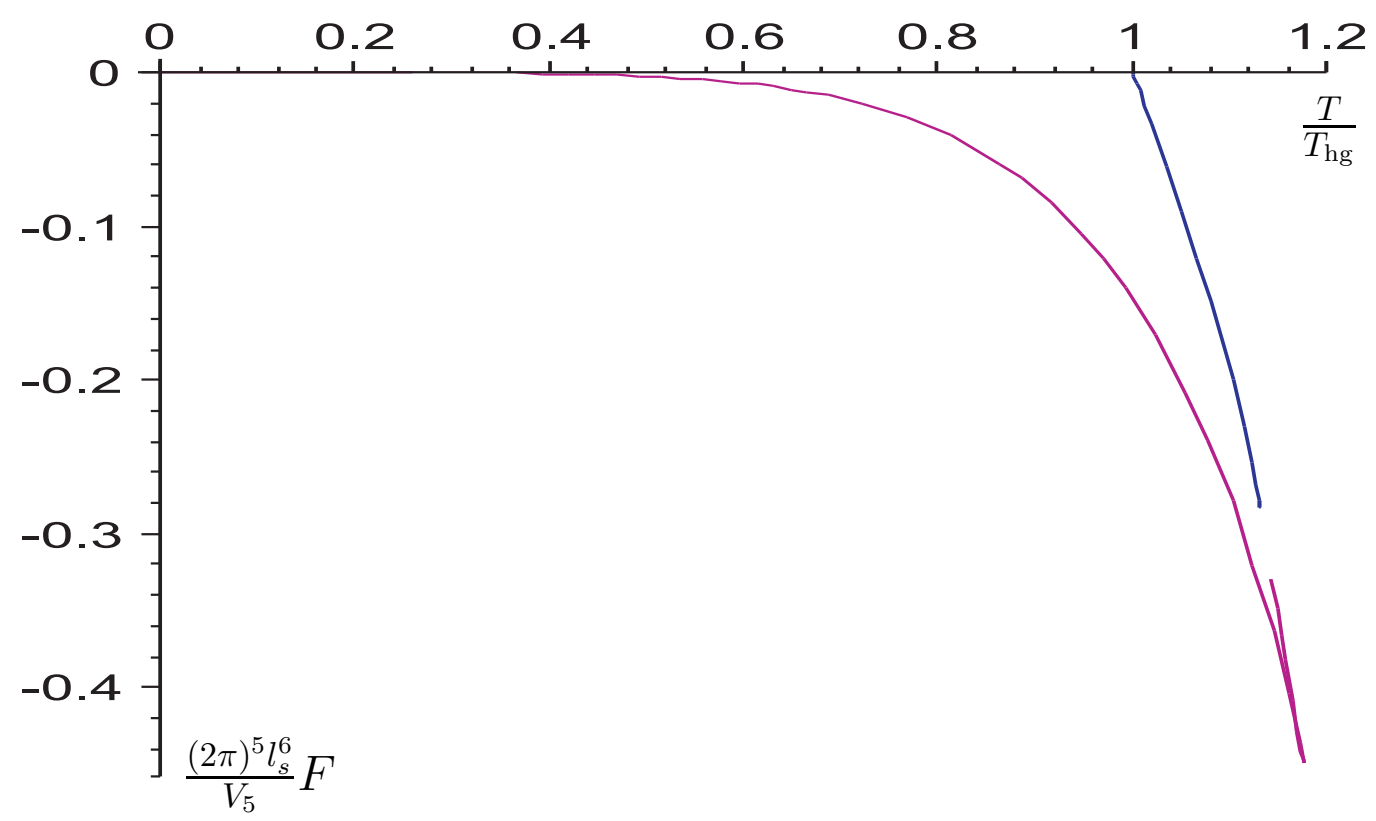

Figure 2: Free energy versus temperature for the near-extremal NS5-branes. Displayed are the non-uniform (blue) and localized (magenta) phases. The uniform phase is located in the point $(T, F)=\left(T_{\mathrm{hg}}, 0\right)$.

Before considering the new data for the localized phase, we first list some important properties of the three different phases (already stated in [7]): ${ }^{10}$

- Uniform phase. This phase corresponds to zero free energy and a fixed temperature $T=T_{\text {hg }}$ (as reviewed in Section 2). It corresponds therefore to a single point in the free energy versus temperature diagram in Figure 2

- Non-uniform phase. This phase starts out in point $(T, F)=\left(T_{\mathrm{hg}}, 0\right)$ and then has increasing temperature and decreasing free energy. It has positive specific heat. For

\footnotetext{
${ }^{10}$ Note that the non-uniform and localized phase have copies with free energy $F^{\prime}=F / k^{2}$ and temperature $T^{\prime}=T(k=2,3, \ldots)$. We do not consider the copied phases here since they are subdominant in this ensemble.
} 
temperatures near the Hagedorn temperature $0 \leq T-T_{\mathrm{hg}} \ll T_{\mathrm{hg}}$, we have [7]

$$
F_{\mathrm{nu}}(T) \simeq-2 \pi V_{5} N^{3} T_{\mathrm{hg}}^{6}\left[1.54 \cdot\left(\frac{T}{T_{\mathrm{hg}}}-1\right)+3.23 \cdot\left(\frac{T}{T_{\mathrm{hg}}}-1\right)^{2}\right] .
$$

This was computed using results of [34].

- Localized phase. The localized phase starts in the point $(T, F)=(0,0)$ which corresponds to the $(2,0)$ SCFT, being an infrared fixed point of the type IIA nearextremal NS5-brane. The first correction to the thermodynamics when moving away from the infrared fixed point by raising the temperature is [7]

$$
F_{\mathrm{loc}}(T)=-\frac{2^{6} \pi^{3}}{3^{7}} V_{5} N^{3} T^{6}\left[1+\frac{2^{5} \zeta(3)}{3^{6}} \frac{T^{6}}{T_{\mathrm{hg}}^{6}}+\mathcal{O}\left(\frac{T^{12}}{T_{\mathrm{hg}}^{12}}\right)\right] .
$$

This was computed using results of [35]. We describe further the thermodynamics of this phase below.

We consider now the localized phase, using the data of [38]. The localized phase starts in the point $(T, F)=(0,0)$ and has decreasing free energy until the maximum temperature $T_{\star}=1.177 \cdot T_{\mathrm{hg}}$ is reached. Then the curve reverses direction and has decreasing temperature and increasing free energy.

The most interesting part of the localized phase is evidently the behavior near the maximal temperature $T_{\star}$. A way to understand the behavior around this point is to consider the temperature versus energy diagram in Fig. 3. The localized phase in the $(E, T)$ diagram is evidently a smooth curve. Therefore, the entropy is continuous for $T \simeq T_{\star}$. However, since the temperature is maximal for $T=T_{\star}$ the $(E, T)$ curve has a horizontal tangent in that point. In terms of the specific heat this means that the localized phase has positive specific heat as $T$ increases from zero to $T_{\star}$, but then reaches infinite specific heat at $T=T_{\star}$. If one continue along the curve in the $(E, T)$ diagram we start from minus infinite specific heat and continue with negative specific heat until the curve has a vertical tangent where the specific heat is zero. Then the specific heat is positive for the remaining part of the curve. ${ }^{11}$

It is interesting to consider more closely the thermodynamics around the critical temperature $T_{\star}$. From the data we get

$$
T \simeq T_{\star}-c\left(E-E_{\star}\right)^{2}, \quad c=0.038, \quad \frac{E_{\star}}{V_{5}}=3.60 \cdot(2 \pi)^{-5} l_{s}^{-6} .
$$

\footnotetext{
${ }^{11}$ In terms of the $(E, r)$ phase diagram the point with infinite specific heat is characterized by the $r$ value for which $E r^{\prime}(E)=1 / 2-r(E)$, while zero specific heat occurs when $r^{\prime}(E)$ is infinite. It is not difficult to see from the phase diagram in Fig. 1 that for each of these two equations there exists one particular solution for $r$ on the localized branch.
} 


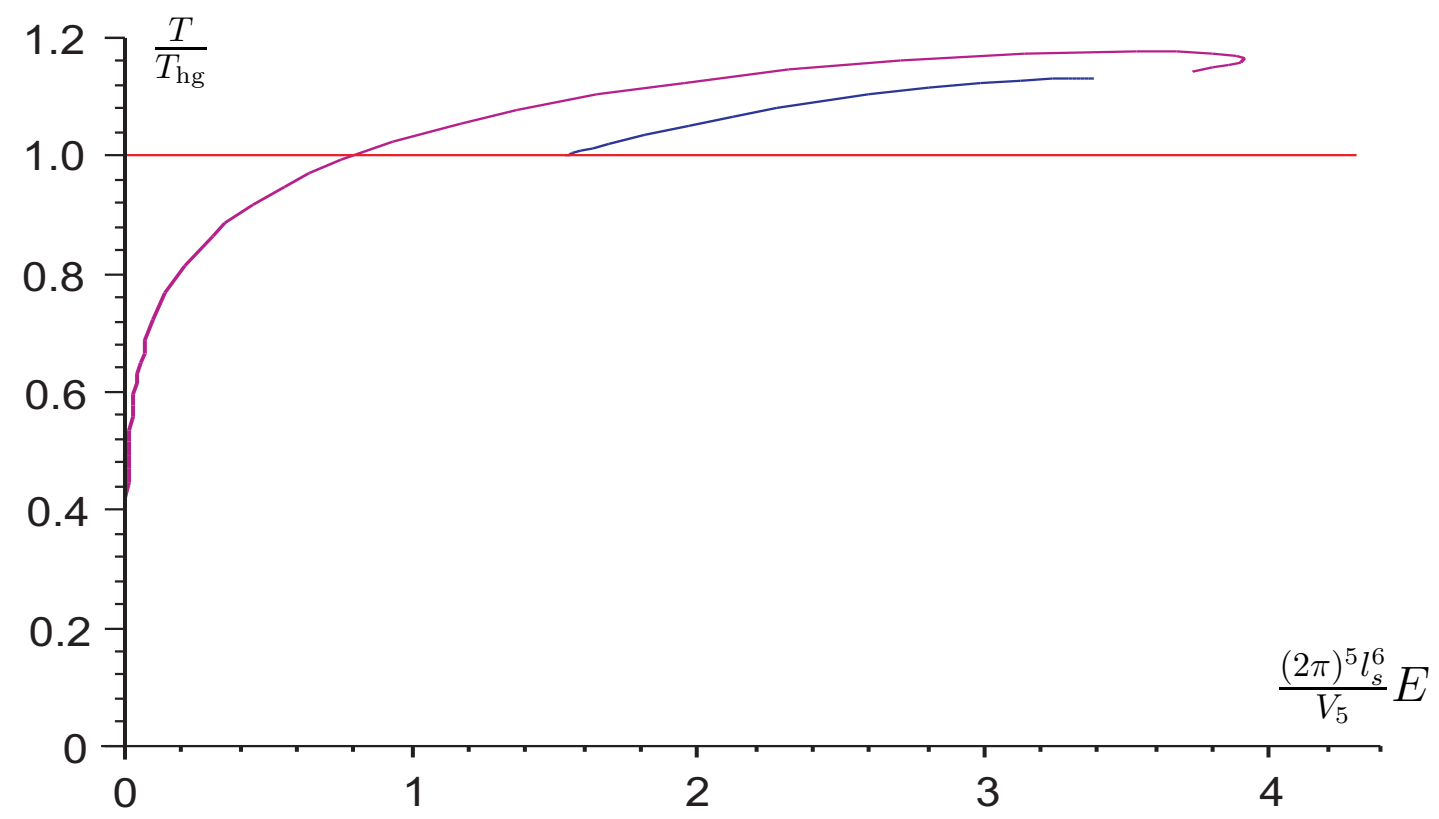

Figure 3: Temperature versus energy for the near-extremal NS5-branes. Displayed are the uniform (red), non-uniform (blue) and localized (magenta) phases.

From this we can obtain the free energy as a function of temperature near the singularity as

$$
\begin{gathered}
F(T) \simeq F_{\star}-S_{\star}\left(T-T_{\star}\right) \pm \frac{2}{3 \sqrt{c} T_{\star}}\left(T-T_{\star}\right)^{3 / 2}, \\
\frac{S_{\star}}{V_{5}}=3.5 \cdot(2 \pi)^{-4} \sqrt{N}, \frac{F_{\star}}{V_{5}} \equiv \frac{1}{V_{5}}\left(E_{\star}-S_{\star} T_{\star}\right)=-0.53 \cdot(2 \pi)^{-5} l_{s}^{-6} .
\end{gathered}
$$

Here the \pm sign corresponds to the two branches on either side of the maximum temperature critical point. In particular, the minus branch is the one that has positive specific heat (so with $E \lesssim E_{\star}$ ) and the plus branch is the one that has negative specific heat (so with $\left.E \gtrsim E_{\star}\right)$. From this expression it is immediately clear that indeed the entropy is finite and continuous at the critical point, while the heat capacity diverges and changes sign.

From Fig. 2 it is clear that there are temperatures where more than one phase is available. To understand what happens for these temperatures, we should first recall that in order for the supergravity solution to be weakly curved we need $N \rightarrow \infty$. Moreover, the free energy for a particular supergravity solution will diverge as $N \rightarrow \infty$. Considering now two phases at the same temperature with free energies $F_{1}$ and $F_{2}$ where $F_{2}>F_{1}$, we can see that the phase with the lowest free energy dominates the Euclidean path integral, since we have that $Z \simeq e^{-\beta F_{1}}+e^{-\beta F_{2}}=e^{-\beta F_{1}}\left(1+e^{-\left(F_{2}-F_{1}\right)}\right) \simeq e^{-\beta F_{1}}$.

Considering then the phase diagram of Fig. 2] we see that we have two phases available when $T_{\mathrm{hg}} \leq T \leq T_{\star}$, but we can conclude from the above that the phase with lowest free energy dominates. We notice that the dominating phase corresponds to the localized phase 
in the energy range $0 \leq E \leq E_{\star}$. This means for example that if one starts in the uniform phase i.e. with zero free energy and temperature $T=T_{\mathrm{hg}}$, then, in the canonical ensemble, the system will decay to the localized solution at the same temperature.

Imagining now that we are in the dominating phase, which is the localized phase as described above, we can consider what happens as we increase the temperature. The heat capacity will increase and eventually becomes infinite once we reach the temperature $T=T_{\star}$. This means that $T_{\star}$ is a limiting temperature. Note that this limiting temperature exceeds the temperature $T_{\mathrm{hg}}$ associated with the uniform phase.

\section{Microcanonical ensemble}

We now turn to the microcanonical ensemble. In Fig. [4 we have displayed the entropy of the three different phases normalized in terms of the entropy of the uniform phase as a function of energy. The three different phases have the following properties: ${ }^{12}$

- Uniform phase. This phase is at the Hagedorn temperature, so the entropy (see (2.2)) is given by

$$
S_{\mathrm{u}}(E)=\frac{E}{T_{\mathrm{hg}}}
$$

and corresponds therefore to the horizontal line in Fig. 4.

- Non-uniform phase. This phase starts out in the point $(E, S)=\left(E_{\mathrm{c}}, S_{\mathrm{c}}\right)$, and for energies close to the critical energy $E_{\mathrm{c}}$ we have [7]

$$
\frac{S(E)}{S_{u}(E)} \simeq 1-0.05 \cdot\left[\frac{(2 \pi)^{5} l_{s}^{6}}{V_{5}}\left(E-E_{\mathrm{c}}\right)\right]^{2}, \frac{E_{\mathrm{c}}}{V_{5}}=1.54 \cdot(2 \pi)^{-5} l_{s}^{-6}, 0 \leq \frac{l_{s}^{6}\left(E-E_{c}\right)}{V_{5}} \ll 1 .
$$

This was computed using results of 34. The entropy function for the entire nonuniform phase, as displayed in Fig. 4] was computed in [7] using the data of [33].

- Localized phase. The localized phase starts out in the point $(E, S)=(0,0)$. At very low energies we have the analytical result [7]

$$
S_{\mathrm{loc}}(E) \simeq \frac{2 \pi^{1 / 3}}{3}\left(\frac{6}{5}\right)^{5 / 6} \frac{\sqrt{N}}{(2 \pi)^{4}} \frac{V_{5}}{l_{s}^{5}}\left((2 \pi)^{5} \frac{l_{s}^{6} E}{V_{5}}\right)^{5 / 6}\left(1+\frac{\zeta(3)}{10 \pi^{2}}(2 \pi)^{5} \frac{l_{s}^{6} E}{V_{5}}\right), \quad \frac{l_{s}^{6} E}{V_{5}} \ll 1 .
$$

This was computed using results of [35]. The entropy function for the entire localized phase, as displayed in Fig. 4, is computed here using the data of [38] and the map of [7.

In the microcanonical ensemble, the phase with the highest entropy dominates. From Fig. 4 we thus conclude that for energies below $E_{\#} / V_{5}=2.51 \cdot(2 \pi)^{-5} l_{s}^{-6}$, the localized

\footnotetext{
${ }^{12}$ Note that the non-uniform and localized phase have copies with entropy $S^{\prime}=S / k^{2}$ and energy $E^{\prime}=$ $E / k^{2}(k=2,3, \ldots)$. We do not consider the copied phases here since they are subdominant in this ensemble.
} 


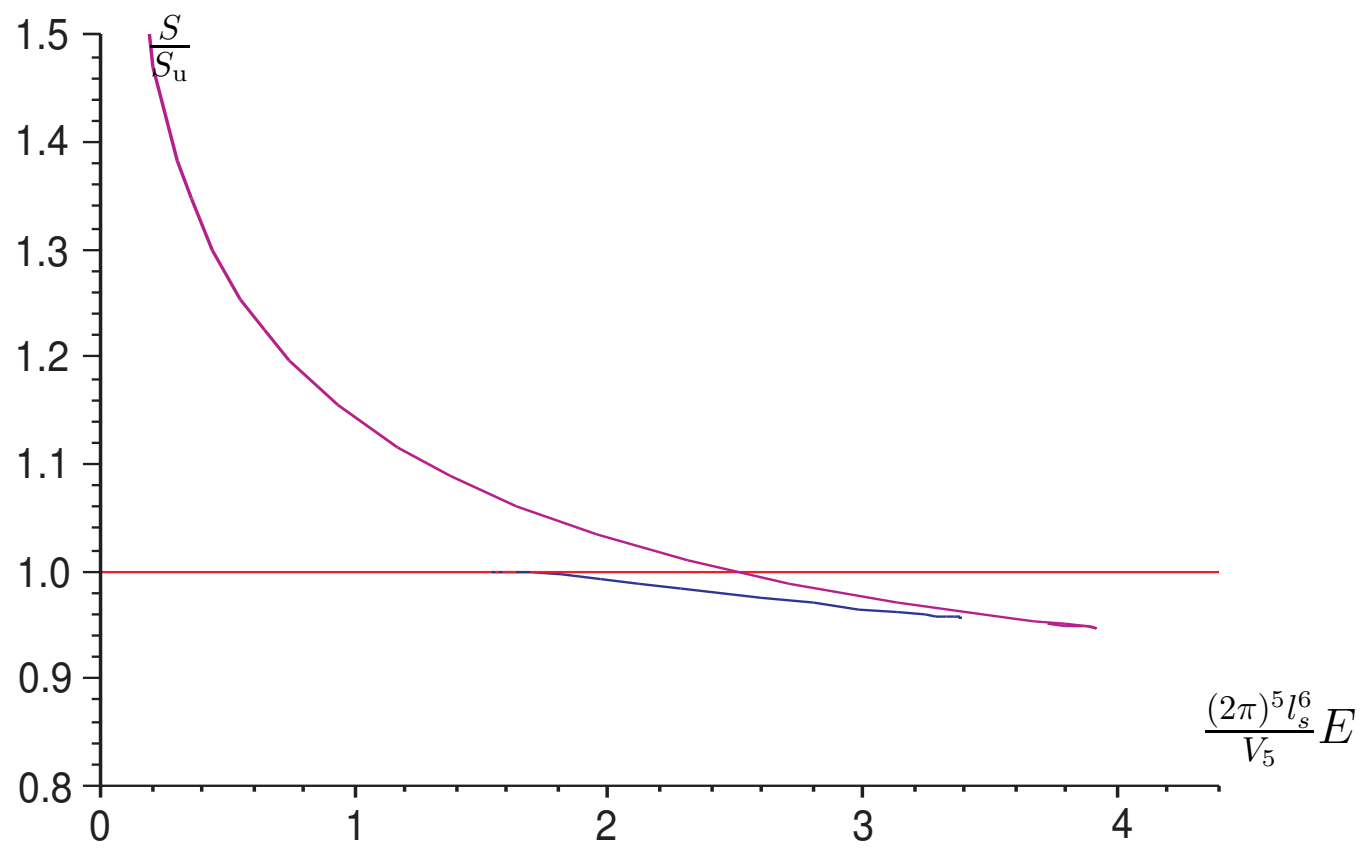

Figure 4: Entropy versus energy for the near-extremal NS5-branes. On the vertical axis we have plotted $S(E) / S_{\mathrm{u}}(E)$ and on the horizontal axis $E / E_{\mathrm{c}}$. Here $S_{\mathrm{u}}(E)$ is the entropy function for the uniform phase of near-extremal NS5-branes. Displayed are the uniform (red), non-uniform (blue) and localized (magenta) phases.

phase is preferred in the canonical ensemble, whereas for energies above that value the system is in the uniform phase. In the range $E_{\#}<E \leq E_{\max }$, with $E_{\max } / V_{5}=3.92$. $(2 \pi)^{-5} l_{s}^{-6}$, we have that the entropy of the uniform phase is greater than that of the localized phase. For $E>E_{\max }$ only the uniform phase is available. Note that $E_{\#}<E_{\star}<$ $E_{\max }$. The non-uniform phase always has lower entropy than the two other phases.

Note that the point of the localized phase in which $E=E_{\max }$ is special. Here we have zero heat capacity, which corresponds to a singular behavior in the microcanonical ensemble. If we imagine an infinitesimal change in the system with energy change $d E$ and a corresponding change in the temperature $d T$, we have that $d T / d E$ diverges as we approach the energy $E_{\max }$. The energy $E_{\max }$ can therefore be seen as a limiting energy for the localized phase in this sense.

It is interesting to consider what happens when throwing in a finite amount of energy $\Delta E$, starting with an energy $E<E_{\#}$. Three things can happen. If $E+\Delta E<E_{\#}$ we stay in the localized phase. If $E_{\#}<E+\Delta E<E_{\max }$ we also jump to a point in the localized phase. However, contrary to before the entropy of the uniform phase at the energy $E+\Delta E$ is greater so the system will go through a phase transition and end up in the uniform phase. Finally, if $E+\Delta E>E_{\max }$ the only available phase is the uniform 
phase, so the system presumably has to end up in that phase.

\section{Comparing the ensembles}

If we compare the canonical and microcanonical ensembles, we see significant differences in the qualitative behavior of the near-extremal NS5-brane thermodynamics. In the canonical ensemble, the localized phase (in the interval given by $E \leq E_{\star}$ ) is always the dominant phase, and it has a positive specific heat and a limiting temperature $T_{\star}$.

Considering instead the microcanonical ensemble, we see that for energies $E \leq E_{\#}$ the localized phase is again the dominant phase, just as in the canonical ensemble. However, the qualitative behavior is very different for energies $E_{\#}<E<E_{\max }$. In that energy range the uniform phase is dominant, and hence we have two different preferred phases in the two ensembles. For energies $E>E_{\max }$ the difference is even more significant since here only the uniform phase is available. For large energies, it thus seems that the two ensembles are completely inequivalent.

Since this system is without gravity one would expect that it always is possible to bring the system in contact with a heat bath of a certain temperature. Therefore, the inequivalence of ensembles could suggest that there is a subtlety in the definition of the canonical ensemble. Alternatively, it could point to the existence of a new hitherto unknown phase of near-extremal NS5-branes, also since this potentially could solve the problem that we seemingly do not have any stable phases with temperatures greater than $T_{\star}$. See the conclusions in Section [6 for further discussion on these issues.

\section{Thermodynamics of $\mathrm{D} p$-brane case}

For comparison, we comment in this section on the thermodynamics for near-extremal $\mathrm{D} p$-branes on a transverse circle. Following [7, this is related to the thermodynamics of phases of Kaluza-Klein black holes in $10-p$ dimensions.

Our statements below concern D0, D1, D2 and D3-branes, which are thus related to phases of Kaluza-Klein black holes in 10, 9, 8 and 7 dimensions. Just as in six dimensions, we know that there exists a uniform black string, non-uniform black string and localized black hole phase. However, contrary to the six-dimensional case, the latter two

branches are not known numerically. Nevertheless, we are still able to make some general observations, based on the results of [7].

From the map of [7] these three phases of neutral Kaluza-Klein black holes generate, just as for the M5-brane case, three corresponding phases of near-extremal $\mathrm{D} p$-branes on a circle: The uniform, non-uniform and localized phase. Moreover, the corresponding curves in the $(E, r)$ phase diagram are believed to be qualitatively of the same form as in that for the NS5-brane given in Fig. 1 However, the thermodynamics is very different in nature, as we now discuss. 
For definiteness, we focus here on $N$ near-extremal D2-branes on a transverse circle, which is dual to finite temperature $\mathrm{N}=4$ supersymmetric $S U(N)$ Yang-Mills on $\mathbb{R}^{2} \times \mathbb{T}^{2}$ (the $\mathbb{T}^{2}$ consists of a compact spatial direction of circumference $\hat{L}$ together with the compact Euclidean time direction). The qualitative picture is expected to hold for the other $\mathrm{D} p$ brane cases as well. The difference with the M5-brane case is best illustrated by looking at the $T(E)$ diagram.

For the uniform phase, consisting of near-extremal D2-branes uniformly smeared on a transverse circle, we have the dependence

$$
T(E) \propto E^{\frac{1}{4}}
$$

i.e. the thermodynamics of near-extremal D3-branes. The uniform phase dominates at high energies. For the localized branch which dominates at low energies we know from [7] that

$$
T(E) \propto E^{\frac{3}{10}}(1+\alpha E), \quad E \ll 1,
$$

with $\alpha<0$ a known constant. Finally, for the non-uniform phase, we know that it emanates from the uniform phase at the critical energy $E_{\mathrm{c}}=0.71 \cdot(2 \pi)^{3} V_{2} N^{2} /\left(\lambda \hat{L}^{3}\right)$. At this critical point we know the ratio of the specific heat of the non-uniform phase and the uniform phase, which is given by [7]

$$
\frac{c_{\mathrm{nu}}}{c_{\mathrm{u}}}=\frac{1}{1+\frac{6}{7} \hat{\gamma} \epsilon_{c}}=0.41
$$

In particular the specific heat in that point is smaller than that of the uniform phase, a feature which holds for any of the $\mathrm{D} p$-brane cases mentioned above.

Based on these results, we have the following features of the three phases in a $T(E)$ diagram:

- The uniform and localized phase start in the origin (with infinite slope) and for very low energies the uniform phase has higher temperature than the localized phase at a given energy. We expect that the localized phase will then cross the uniform phase already for relatively low energies, at least below the critical energy where the non-uniform phase starts.

- Since the slope in the $T(E)$ diagram is the inverse of the specific heat, it follows from (5.3) that the slope of the non-uniform phase at the critical point $(E, T)=\left(E_{\mathrm{c}}, T_{\mathrm{c}}\right)$ is larger than that of the uniform phase.

- We assume that the localized and non-uniform branch meet in a qualitatively similar way, as observed explicitly in Fig. 3 for the NS5-brane.

We are then led to a picture that is summarized in the $T(E)$ diagram of Fig. 5 ,

We note that the picture of the thermodynamics displayed in Fig. 15 implies that the $(E, r)$ phase diagram for near-extremal $\mathrm{D} p$-branes on a transverse circle is qualitatively 


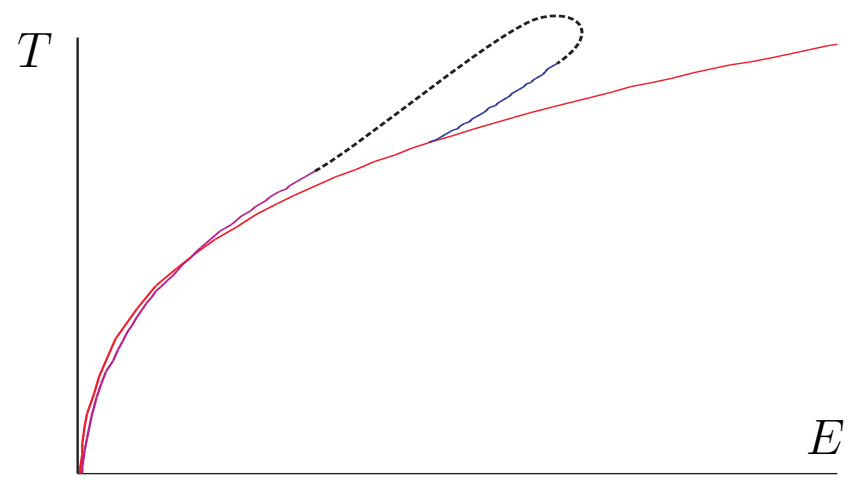

Figure 5: Qualitative picture of energy versus temperature for the phases of near-extremal $\mathrm{D} p$-branes on a transverse circle. Shown are the uniform (red), non-uniform (blue) and localized (magenta) phases. The dashed curve connects the localized and non-uniform phase.

of the same form as that for the NS5-brane in Fig. 1. This means in particular that as the energy increases from zero in the localized phase we have that: i) First the point is reached where the specific heat becomes infinite. Here the maximal temperature of the localized phase occurs; ii) Increasing the energy further one reaches the point where the specific heat is zero. Here the maximal energy of this phase occurs. ${ }^{13}$

Hence, just as in the NS5-brane case, the localized phase has a maximal temperature. On the other hand, contrary to NS5-brane case, where the uniform phase has the constant Hagedorn temperature, the uniform phase exists for any temperature. As a consequence there is no limiting temperature in the system when considering near-extremal $\mathrm{D} p$-branes on a circle. In particular, for sufficiently high temperatures the system will be in the uniform phase. Below some critical temperature the localized phase will be favored. Moreover, the canonical and microcanonical ensemble show the same behavior.

\section{Conclusions}

We have examined the thermodynamics of the near-extremal type IIA NS5-brane using its description as an M5-brane with a transverse circle, and the relation of the latter to the phases of six-dimensional Kaluza-Klein black holes. We have found that in the canonical ensemble there is a new background of near-extremal M5-branes localized on the transverse circle, which dominates over the usual near-extremal NS5-brane background. This new phase has the surprising feature that it has a limiting temperature $T_{\star}=1.177 \cdot T_{\mathrm{hg}}$, which lies above the Hagedorn temperature $T_{\mathrm{hg}}$ of the usual NS5-brane phase. We have also

\footnotetext{
${ }^{13}$ The conditions for infinite and zero specific heat are in this case $E r^{\prime}(E)=(5-p) / 2-r(E)$ and $1 / r^{\prime}(E)=0$ respectively.
} 
considered the microcanonical ensemble and compared it with the canonical ensemble, indicating some significant differences between the two.

We conclude with the following remarks:

High energy regime versus low energy regime. The analysis of the type IIA NS5-brane thermodynamics of this paper is valid in the low energy regime $E \ll N V_{5} l_{s}^{-6}$. This is the regime in which the effective string coupling is sufficiently large to see the non-perturbative effects of the eleven-dimensional circle. In the high energy regime $E \gg$ $N V_{5} l_{s}^{-6}$ we instead have that the effective string coupling is small. In fact, the nearextremal NS5-brane background has an exact worldsheet CFT description which is asymptotically free in the sense that we can define a perturbation theory around infinite energy in powers of $1 / E$ [24]. The results of [12] then suggest that the near-extremal type IIA NS5-brane has a high-energy phase that has $T>T_{\text {hg }}$ and negative specific heat. In figure [6] we have made a sketch of this phase in an $E$ versus $T$ diagram (see [23] for a similar sketch) together with the phases described in this paper as displayed in Figure $3^{14}$

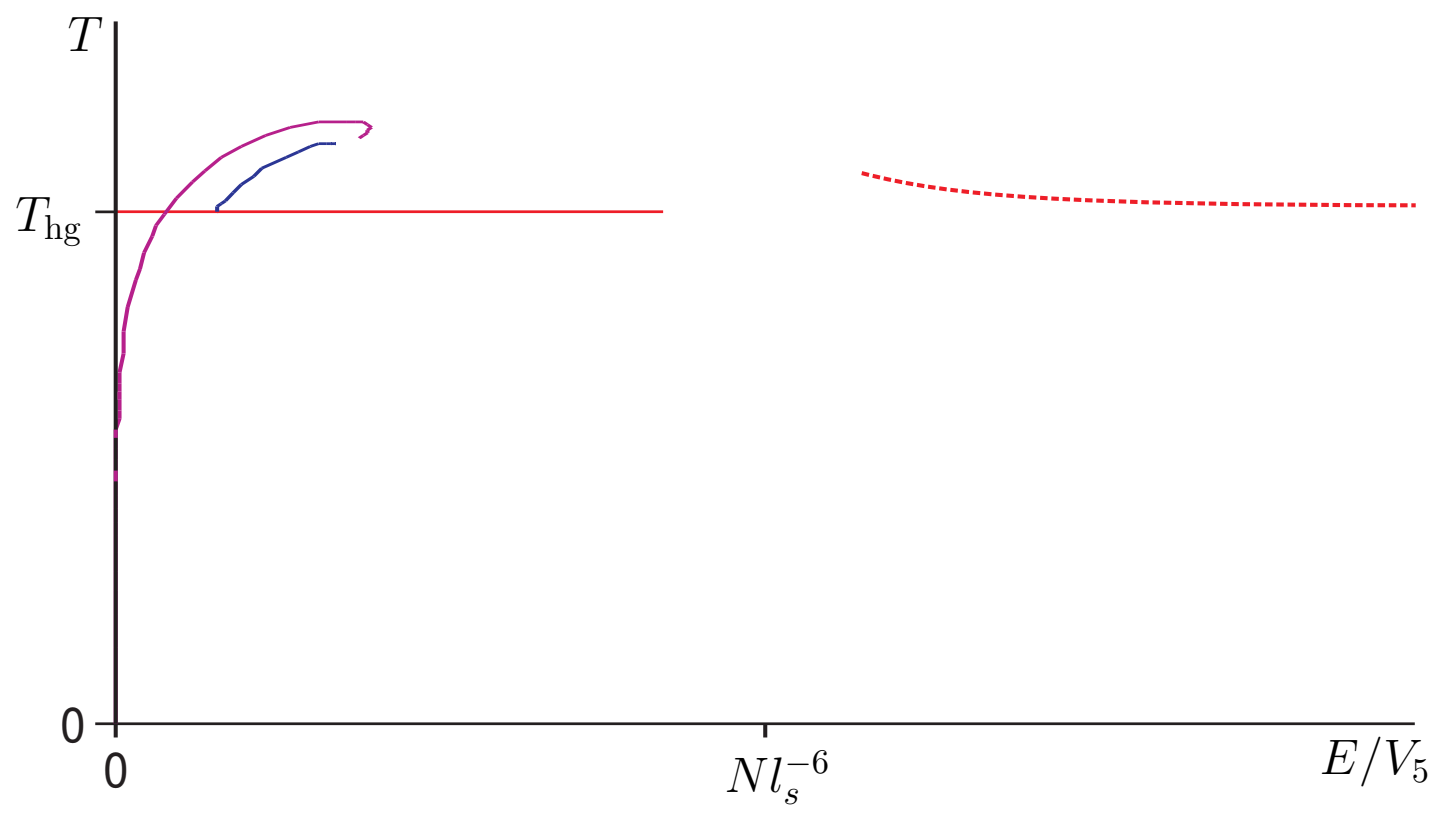

Figure 6: Temperature versus energy for the near-extremal type IIA NS5-brane. Displayed are the same phases as in Figure 3, but in addition we have sketched the high energy phase of the near-extremal NS5-brane.

Now, the results of this paper are all concerned with the low energy regime $E \ll$ $N V_{5} l_{s}^{-6}$. This is the regime in which the effective coupling of the exact worldsheet CFT description for the type IIA NS5-brane background is strong. We see thus that in the

\footnotetext{
${ }^{14}$ According to Refs. 44 45] the instability of the high energy thermodynamics of near-extremal NS5branes found in [12] should be holographically dual to a Gregory-Laflamme like instability, corresponding to longitudinal perturbations of the NS5-brane. This is in contrast to Ref. [12] where it is argued that the instability is of stringy origin.
} 
microcanonical ensemble there are no discrepancies or problems with the existence of the new phases of this paper, since we have a clear distinction between the low energy and high energy regimes. The problems only appear when going to the canonical ensemble. If we start at very low temperatures and heat up the type IIA near-extremal NS5-brane, we will be in the localized phase all the way up to the temperature $T_{\star}$, which lies above $T_{\mathrm{hg}}$. Here the canonical ensemble seems well-defined, as the localized phase of near-extremal M5-branes clearly does not have large fluctuations around it. On the other hand, seen from the point of view of the high energy regime, it is possible that the fluctuations of the energy are large which seems to suggest that the canonical ensemble is ill-defined near the temperature $T_{\mathrm{hg}}$. We thus have an apparent discrepancy. However, in our opinion this discrepancy cannot mean that the canonical ensemble is ill-defined as one heats up the system from zero temperature to $T_{\star}$. This is because such a process is clearly described by classical solutions and we know that fluctuations around these solutions are suppressed, which means that we have a well-defined temperature. Hence, the discrepancy must have another source. We think that this should be very interesting to examine further.

Near-extremal NS5-brane phases with bubbles. In this paper, we have focussed on the type IIA NS5-brane phases that can be obtained from six-dimensional KaluzaKlein black holes without Kaluza-Klein bubbles. However, there exists a large class of six-dimensional bubble-black hole sequences [14 that contain both event horizons and Kaluza-Klein bubbles. These can be mapped to corresponding phases of near-extremal NS5-branes involving Kaluza-Klein bubbles [15]. For the purpose of this paper, these are not relevant as they are subdominant in both the canonical and the microcanonical ensemble. In particular, in the canonical ensemble one finds that the NS5-brane/bubble phases have positive free energy, while we also note that the temperatures of these phases approach the Hagedorn temperature of the uniform phase in the limit where the size of the bubble goes to zero. In the microcanonical ensemble one observes that the entropy of the NS5-brane/bubble phases lies below that of the uniform phase, and approaches the Hagedorn behavior $S \simeq E / T_{\mathrm{hg}}$ of the uniform phase in the limit where the size of the bubble goes to zero. Finally, it is amusing to note that in many of the phases involving Kaluza-Klein bubbles one can have arbitrarily high temperatures.

Putting fluxes on the type IIA NS5-brane. One can deform the near-extremal NS5-brane by adding a D0, D2 or D4-brane flux on the world-volume. The dual theories on the world-volume of the NS5-brane are the OD-theories [46, 47]. These should be possible to study using the techniques of this paper. This could be interesting in view of the recently studied gravitational and thermodynamic instabilities [48, 49] of these types of brane bound states.

Type IIB NS5-brane. An obvious question to address is if our results of this paper on the thermodynamics of the type IIA NS5-brane also can be used to say anything about the type IIB NS5-brane, since these are related by a longitudinal T-duality. However, if we want to take a decompactification limit of the T-dual circle on the IIB side, it corresponds 
to a circle of string size at the IIA side, and thus we cannot use supergravity on the IIA side to predict the behavior on the IIB side. In particular, the new localized phase for the type IIA NS5-brane obtained in this paper cannot be related to a corresponding phase for the (decompactified) type IIB NS5-brane. Therefore, it seems that a separate study is required for the thermodynamics of the near-extremal type IIB NS5-brane. Another way to understand this is to notice that the low energy behavior of the type IIB near-extremal NS5-brane is given by a six-dimensional super-Yang-Mills theory. Therefore, one would have to understand the low energy behavior using gauge theory rather than gravity.

\section{Acknowledgments}

We thank Micha Berkooz, Jan de Boer, Poul Henrik Damgaard, Vasilis Niarchos and Peter Orland for useful discussions. Special thanks to Hideaki Kudoh and Toby Wiseman for explanations and providing us with the numerical results of Ref. 38. Work partially supported by the European Community's Human Potential Programme under contract MRTN-CT-2004-005104 'Constituents, fundamental forces and symmetries of the universe'.

\section{References}

[1] N. Seiberg, "New theories in six-dimensions and matrix description of M theory on $T^{5}$ and $T^{5} / Z_{2}, "$ Phys. Lett. B408 (1997) 98-104, hep-th/9705221.

[2] M. Berkooz, M. Rozali, and N. Seiberg, "Matrix description of M theory on $T^{4}$ and $T^{5}, "$ Phys. Lett. B408 (1997) 105-110, hep-th/9704089.

[3] R. Dijkgraaf, E. Verlinde, and H. Verlinde, "Notes on matrix and micro strings," Nucl. Phys. Proc. Suppl. 62 (1998) 348, hep-th/9709107.

[4] N. Seiberg, "Notes on theories with 16 supercharges," Nucl. Phys. Proc. Suppl. 67 (1998) 158-171, hep-th/9705117.

[5] J. M. Maldacena and C. Nunez, "Towards the large $N$ limit of pure $\mathrm{N}=1$ super yang mills," Phys. Rev. Lett. 86 (2001) 588-591, hep-th/0008001

[6] T. Harmark and N. A. Obers, "Black holes on cylinders," JHEP 05 (2002) 032, hep-th/0204047.

[7] T. Harmark and N. A. Obers, "New phases of near-extremal branes on a circle," JHEP 09 (2004) 022, hep-th/0407094.

[8] J. M. Maldacena, "Statistical entropy of near extremal five-branes," Nucl. Phys. B477 (1996) 168-174, hep-th/9605016 
[9] J. M. Maldacena and A. Strominger, "Semiclassical decay of near-extremal fivebranes," JHEP 12 (1997) 008, hep-th/9710014

[10] T. Harmark and N. A. Obers, "Hagedorn behaviour of little string theory from string corrections to NS5-branes," Phys. Lett. B485 (2000) 285, hep-th/0005021

[11] M. Berkooz and M. Rozali, "Near Hagedorn dynamics of NS fivebranes, or a new universality class of coiled strings," JHEP 05 (2000) 040, hep-th/0005047.

[12] D. Kutasov and D. A. Sahakyan, "Comments on the thermodynamics of little string theory," hep-th/0012258

[13] O. Aharony, A. Giveon, and D. Kutasov, "LSZ in LST," Nucl. Phys. B691 (2004) $3-78$, hep-th/0404016

[14] H. Elvang, T. Harmark, and N. A. Obers, "Sequences of bubbles and holes: New phases of Kaluza-Klein black holes," JHEP 01 (2005) 003, hep-th/0407050.

[15] T. Harmark and N. A. Obers. Work in progress.

[16] O. Aharony, M. Berkooz, D. Kutasov, and N. Seiberg, "Linear dilatons, NS5-branes and holography," JHEP 10 (1998) 004, hep-th/9808149

[17] A. Giveon and D. Kutasov, "Little string theory in a double scaling limit," JHEP 10 (1999) 034, hep-th/9909110

[18] A. Giveon and D. Kutasov, "Comments on double scaled little string theory," JHEP 01 (2000) 023, hep-th/9911039

[19] O. Aharony, "A brief review of 'little string theories'," Class. Quant. Grav. 17 (2000) 929, hep-th/9911147.

[20] K. Narayan and M. Rangamani, "Hot little string correlators: A view from supergravity," JHEP 08 (2001) 054, hep-th/0107111.

[21] P. A. DeBoer and M. Rozali, "Thermal correlators in little string theory," Phys. Rev. D67 (2003) 086009, hep-th/0301059

[22] O. Aharony, B. Fiol, D. Kutasov, and D. A. Sahakyan, "Little string theory and heterotic/type II duality," Nucl. Phys. B679 (2004) 3-65, hep-th/0310197.

[23] A. Parnachev and A. Starinets, "The silence of the little strings," hep-th/0506144.

[24] D. Kutasov, "Introduction to little string theory,". Prepared for ICTP Spring School on Superstrings and Related Matters, Trieste, Italy, 2-10 Apr 2001.

[25] T. Harmark and N. A. Obers, "Black holes and black strings on cylinders," Fortsch. Phys. 51 (2003) 793-798, hep-th/0301020. 
[26] T. Harmark and N. A. Obers, "New phases of thermal SYM and LST from Kaluza-Klein black holes," Fortsch. Phys. 53 (2005) 536-541, hep-th/0503021.

[27] T. Harmark and N. A. Obers, "Phases of Kaluza-Klein black holes: A brief review," hep-th/0503020.

[28] B. Kol, "The phase transition between caged black holes and black strings: A review," hep-th/0411240

[29] T. Wiseman, "From black strings to black holes," Class. Quant. Grav. 20 (2003) 1177-1186, hep-th/0211028.

[30] T. Harmark and N. A. Obers, "Phase structure of black holes and strings on cylinders," Nucl. Phys. B684 (2004) 183-208, hep-th/0309230

[31] T. Harmark and N. A. Obers, "General definition of gravitational tension," JHEP 05 (2004) 043, hep-th/0403103

[32] T. Harmark and N. A. Obers, "New phase diagram for black holes and strings on cylinders," Class. Quantum Grav. 21 (2004) 1709-1724, hep-th/0309116.

[33] T. Wiseman, "Static axisymmetric vacuum solutions and non-uniform black strings," Class. Quant. Grav. 20 (2003) 1137-1176, hep-th/0209051.

[34] E. Sorkin, "A critical dimension in the black-string phase transition," Phys. Rev. Lett. 93 (2004) 031601, hep-th/0402216

[35] T. Harmark, "Small black holes on cylinders," Phys. Rev. D69 (2004) 104015, hep-th/0310259.

[36] D. Gorbonos and B. Kol, "A dialogue of multipoles: Matched asymptotic expansion for caged black holes," JHEP 06 (2004) 053, hep-th/0406002

[37] D. Gorbonos and B. Kol, "Matched asymptotic expansion for caged black holes: Regularization of the post-Newtonian order," hep-th/0505009.

[38] H. Kudoh and T. Wiseman, "Connecting black holes and black strings," Phys. Rev. Lett. 94 (2005) 161102, hep-th/0409111.

[39] G. T. Horowitz, "Playing with black strings," hep-th/0205069.

[40] P. Bostock and S. F. Ross, "Smeared branes and the Gubser-Mitra conjecture," Phys. Rev. D70 (2004) 064014, hep-th/0405026.

[41] O. Aharony, J. Marsano, S. Minwalla, and T. Wiseman, "Black hole - black string phase transitions in thermal $1+1$ dimensional supersymmetric Yang-Mills theory on a circle," Class. Quant. Grav. 21 (2004) 5169-5192, hep-th/0406210. 
[42] H. Kudoh and U. Miyamoto, "On non-uniform smeared black branes," hep-th/0506019.

[43] T. Harmark, V. Niarchos, and N. A. Obers, "Instabilities of near-extremal smeared branes and the correlated stability conjecture," JHEP 010 (2005) 045, hep-th/0509011.

[44] M. Rangamani, "Little string thermodynamics," JHEP 06 (2001) 042, hep-th/0104125.

[45] A. Buchel, "On the thermodynamic instability of LST," hep-th/0107102.

[46] T. Harmark, "Open branes in space-time non-commutative little string theory," Nucl. Phys. B593 (2001) 76-98, hep-th/0007147.

[47] R. Gopakumar, S. Minwalla, N. Seiberg, and A. Strominger, "OM theory in diverse dimensions," JHEP 08 (2000) 008, hep-th/0006062.

[48] J. J. Friess and S. S. Gubser, "Instabilities of D-brane bound states and their related theories," hep-th/0503193.

[49] S. F. Ross and T. Wiseman, "Smeared D0 charge and the Gubser-Mitra conjecture," Class. Quant. Grav. 22 (2005) 2933-2946, hep-th/0503152 